\title{
Produção de farinha de banana verde (Musa spp.) para aplicação em pão de trigo integral
}

\author{
Production of unripe banana flour (Musa spp) for application in whole wheat bread
}

\author{
Bruna Andina Andrade ${ }^{1}$, Dóris Back Perius ${ }^{1 *}$, Natália Vergara de Mattos", Márcia de Mello Luvielmo, \\ Myriam Salas Mellado' \\ Universidade Federal do Rio Grande (FURG), Escola de Química e Alimentos, Laboratório de Tecnologia e Alimentos (LTA), Rio Grande/RS - Brasil
}

\author{
*Corresponding Author \\ Dóris Back Perius, Universidade Federal do Rio Grande (FURG), Escola de Química e Alimentos, Laboratório de Tecnologia e Alimentos (LTA), \\ Campus Carreiros, Av. Itália, km 8, CEP: 96203-900, Rio Grande/RS - Brasil, e-mail: doh_perius@hotmail.com
}

Cite as: Production of unripe banana flour (Musa spp) for application in whole wheat bread. Braz. J. Food Technol., v. 21, e2016055, 2018.

Received: May 06, 2016; Accepted: Sept. 01, 2017

\section{Resumo}

A farinha de banana verde (FBV) é uma alternativa viável para o aproveitamento dos frutos e possui alto teor de amido resistente (AR), que apresenta papel fisiológico similar ao das fibras alimentares. O objetivo deste trabalho foi produzir FBV de dois genótipos de bananeira (Prata e Caturra), além de selecionar aquele com maior teor de AR, para posterior substituição de parte da farinha de trigo integral por FBV, em uma formulação de pão de fôrma integral. Para a produção das farinhas, os frutos foram descascados, cortados em rodelas e estas foram submersas em uma solução de ácido ascórbico e ácido cítrico, sendo então distribuídas em bandejas e secas a $50^{\circ} \mathrm{C}$, por 7 horas, e moídas. As farinhas foram submetidas às análises de composição proximal e determinação do teor de AR. Posteriormente, foram processados pães integrais com a substituição de $10 \%, 15 \%$ e $20 \%$ de farinha de trigo integral pela FBV que obteve o maior teor de AR. Os pães controle e aquele que apresentou maior teor de AR e boas características tecnológicas, como volume específico, dureza e cor, foram submetidos aos testes sensoriais de aceitação e intenção de compra. O teor de AR encontrado para as FBV Prata e Caturra foram 24,1\% e 13,7\%, respectivamente. O pão com substituição de 15\% de farinha de trigo integral por FBV foi escolhido para a análise sensorial. As formulações controle e com 15\% de FBV atingiram Índices de Aceitação Sensorial de 88,7\% e 82,1\%, respectivamente. A atitude dos avaliadores foi positiva, segundo a qual $56 \%$ dos avaliadores "comprariam frequentemente" ou "comprariam sempre" o pão com 15\% de FBV, não tendo sido observada rejeição para nenhuma das formulações.

Palavras-chave: Amido resistente; Características tecnológicas; Sensorial; Panificação.

\section{Abstract}

Unripe banana flour (UBF) is a viable alternative for using the fruit, since it has a high resistant starch (RS) content which presents a physiological role similar to that of dietary fibre. The objective of this study was to produce UBF from two banana genotypes (Lady Finger and Cavendish), and select the one with the highest level of RS for subsequent replacement of part of the whole wheat flour by UBF in a formulation for whole wheat loaves. For the production of UBF, the fruits were peeled, cut into slices, submerged in a solution of ascorbic acid and citric acid, spread on trays, dried at $50{ }^{\circ} \mathrm{C}$ for 7 hours and then ground. The flours were submitted to proximate composition and RS content analyses. Whole wheat loaves were then processed, replacing $10 \%, 15 \%$ and $20 \%$ of the whole wheat flour by the UBF containing the highest resistant starch content. The control loaves and the one with the highest RS content and good technological characteristics, such as specific volume, hardness and colour, were submitted to a sensory test for acceptance and purchase intent. The resistant starch contents found in the Finger and Cavendish UBF samples were $24.1 \%$ and $13.7 \%$ respectively. Loaves with $15 \%$ substitution of the whole wheat flour by UBF were chosen for the sensory analysis. The Control loaf formulation and that with $15 \%$ UBF reached acceptance levels of $88.7 \%$ and $82.1 \%$, respectively. The attitude of the judges was positive since $56 \%$ of them answered they would buy the bread with 15\% UBF frequently or always, and none of the formulations were rejected.

Keywords: Resistant starch; Technological characteristics; Sensory; Baking. 


\section{Introdução}

A importância do cultivo da banana está relacionada ao fato de esta ser um fruto nutritivo e acessível à maior parte da população (SOUZA et al., 2011). Ademais, a facilidade de proliferação e o manejo fazem com que esta fruta apresente um destaque econômico e social, sendo mundialmente comercializada, principalmente nas regiões tropicais (COELHO JÚNIOR, 2013).

A banana destaca-se na primeira posição no ranking mundial das frutas, sendo o Brasil o produtor de aproximadamente sete milhões de toneladas, em uma área de 489.937 hectares. O país apresenta condições favoráveis para o seu cultivo e, por esse motivo, a banana é cultivada na maioria dos Estados brasileiros (IBGE, 2016).

A banana é classificada como um fruto climatérico, altamente perecível, que apresenta alta taxa respiratória e alta produção de etileno após a colheita (PRILL et al., 2012). As perdas quantitativas e qualitativas em virtude de sua perecibilidade são significativas. Do total de bananas colhidas, cerca de $40 \%$ a $50 \%$ chegam, de fato, aos consumidores (MEDINA; PEREIRA, 2004).

Uma alternativa é a industrialização da banana ainda verde, na forma de farinha, a qual reduzirá as perdas pós-colheita e durante o transporte (SARAWONG et al., 2014). O fruto pode ser processado em diferentes estágios de maturação, mas, para a obtenção da farinha, é necessário processá-la completamente verde (ORMENESE, 2010).

$O$ amido é o mais importante componente da farinha de banana verde (FBV), representando uma fração mássica de 70 a $80 \mathrm{~g}$ de amido total/100 g de produto. De acordo com Juarez-Garcia et al. (2006), a FBV produzida sob condições específicas é composta de cerca de 73,4 g de amido total/100 $\mathrm{g}$ de produto.

O amido pode ser classificado como glicêmico ou resistente. $\mathrm{O}$ amido glicêmico é degradado à glicose por enzimas, no trato digestivo. Já o amido resistente (AR) é aquele que resiste à digestão no intestino delgado, mas é fermentado, no intestino grosso, pela microflora bacteriana (YUE; WARING, 1998). Pode-se dizer que o amido resistente é a fração que não fornecerá glicose ao organismo, mas que será fermentada no intestino grosso para produzir gases e ácidos graxos de cadeia curta, principalmente. Devido a esta característica, considera-se que os efeitos do AR sejam, em alguns casos, comparáveis aos da fibra alimentar (CHAMP; FAISANT, 1996).

A utilização da FBV é muito vantajosa pelo seu elevador teor de AR, compostos fenólicos e antioxidantes (PEREIRA, 2007; SILVA et al., 2015).

O consumo de farinha integral é encorajado principalmente pelo alto teor de fibras, com comprovados benefícios à saúde humana; porém, sensorialmente, a farinha integral confere características não desejadas ao produto. O AR, além de funcionar como fibra alimentar, altera menos o produto convencional, possibilitando incluir funcionalidade sem prejudicar muito as características tecnológicas e sensoriais.

Além disso, grande parte do AR encontrado na FBV é do tipo 2, cuja cristalinidade o torna mais resistente à hidrólise e, por consequência, com feitos positivos para o cólon e implicações benéficas para a saúde humana (LANGKILDE et al., 2002), com redução dos níveis glicêmicos e insulinêmicos, quando ingerido.

Este trabalho teve como objetivo produzir FBV a partir de dois genótipos de bananeira (Prata e Caturra), além de selecionar o genótipo com maior teor de AR, substituir parte da farinha de trigo integral por FBV em uma formulação de pão de fôrma integral e avaliar sensorialmente o produto.

\section{Material e métodos}

\subsection{Material}

O estudo foi realizado com bananas (Musa spp.) das cultivares Prata e Caturra, que foram adquiridas na Central de Hortigranjeiros da cidade do Rio Grande-RS/Brasil. As amostras foram coletadas, em uma mesma data, antes de entrarem para a câmara de maturação. A escolha da matéria-prima foi feita a partir da coloração da casca, tendo sido escolhidas somente bananas totalmente verdes, conforme a escala de maturação descrita por Chitarra e Chitarra (2005).

A matéria-prima utilizada para elaboração dos pães foi farinha de trigo integral adquirida no comércio local, assim como os demais ingredientes utilizados para produção dos pães, como gordura vegetal hidrogenada, cloreto de sódio e sacarose. Foram utilizados também ácido ascórbico P.A. da marca Vetec e fermento biológico fresco.

\subsection{Métodos}

O estudo foi desenvolvido no Laboratório de Tecnologia de Alimentos (LTA) da Universidade Federal do Rio Grande.

\subsubsection{Obtenção da farinha de banana verde}

Os frutos com casca foram lavados com água corrente, imersos em água clorada 50 ppm por 10 minutos, novamente imersos em água clorada 20 ppm por 10 minutos, enxaguados e descascados manualmente. Em seguida, a fruta foi cortada em rodelas de aproximadamente três milímetros de espessura, sendo estas submersas em uma solução contendo ácido ascórbico (0,35 g/L) e ácido cítrico (5 g/L), por 15 minutos. As rodelas de banana foram dispostas em bandejas perfuradas e secas em forno (G.PANIZ, modelo FTE 150), com circulação de ar na temperatura de $50{ }^{\circ} \mathrm{C}$, por 7 horas. As rodelas de banana, após sua secagem, foram trituradas em liquidificador de facas duplas (marca Pick-liq) e peneiradas em peneira de abertura de 42 mesh. A farinha obtida foi acondicionada 
Produção de farinha de banana verde (Musa spp.) para aplicação em pão de trigo integral

Andrade, B. A. et al.

em sacos de polietileno de baixa densidade e armazenada em refrigeração.

\subsubsection{Rendimento das farinhas}

O rendimento das farinhas foi determinado de acordo com a Equação 1, descrita por Santos et al. (2010).

$R=\frac{F}{P} \times 100$

em que: $\mathrm{R}=$ rendimento $(\%) ; \mathrm{F}$ = quantidade de farinha obtida (g); $\mathrm{P}$ = quantidade de polpa de banana verde (g).

\subsubsection{Formulação e elaboração dos pães integrais}

Os pães integrais do tipo fôrma foram preparados a partir das formulações apresentadas na Tabela 1. Para a formulação controle, foram utilizados $300 \mathrm{~g}$ de farinha de trigo integral. Nas formulações com FBV, a farinha de trigo integral foi substituída em 10, 15 e 20\% por FBV.

Os pães de fôrma foram preparados pelo método direto. Inicialmente, foram misturados os ingredientes secos em uma batedeira (KITCHENAID, modelo BEA30ABAIM) em velocidade baixa, durante 3 minutos. Em seguida, foram acrescentados os ingredientes água, gordura e fermento biológico, misturando-se até obtenção de uma massa consistente. A massa foi deixada em descanso por 10 minutos, sendo então cortada em pedaços de $165 \mathrm{~g}$. Os pedaços foram boleados e moldados até formar bisnagas. Cada bisnaga foi colocada em uma fôrma metálica e levada à estufa de fermentação, em posições alternadas (QUIMIS, modelo Q31711-43), e permaneceram a $30{ }^{\circ} \mathrm{C}$ e $80 \%$ UR, durante 1 hora e 30 minutos. As triplicatas foram forneadas em forno elétrico (FISCHER, modelo Diplomata) a $200{ }^{\circ} \mathrm{C}$, por 20 minutos. A avaliação tecnológica foi realizada após 1 hora de resfriamento.

\subsubsection{Avaliação física}

Foi calculada a perda por cocção (\% PC) através da Equação 2, descrita por Paz et al. (2015). Este cálculo foi realizado para as quatro formulações.

$P C(\%)=\frac{M_{\text {massa }}-M_{\text {pão }}}{M_{\text {massa }}} .100$

em que: $M_{\text {massa }}=$ massa de massa; $M_{\text {pão }}=$ massa de pão.

\subsubsection{Avaliação tecnológica dos pães}

O volume específico $(\mathrm{mL} / \mathrm{g})$ foi obtido pela razão entre o volume aparente $(\mathrm{mL})$, realizado por deslocamento de sementes de painço, segundo Pizzinatto et al. (1993), e a massa (g), após forneamento. A dureza do miolo foi medida no pão fresco, após 1 hora de forneamento, sendo realizada em texturômetro TA-XT2 (Stable Micro Systems, UK), método n. 74-09.01, de acordo com AACC (2000). A determinação da cor do miolo e da crosta foi realizada em colorímetro marca Minolta ${ }^{\circledR}$ modelo CR 400, avaliando $L^{*}$ (luminosidade) e $a^{*}$ e b* (coordenadas de cromaticidade). Foram realizadas cinco medidas em cada unidade de pão, de acordo com Minolta Corporation (1994).

\subsubsection{Composição proximal das FBV e pães de fôrma integral}

A umidade foi determinada pelo método n. ${ }^{\circ}$ 935.29, de acordo com AOAC (HORWITZ, 2000); o teor de proteína foi determinado pelo método de microKjeldahl, n. ${ }^{\circ}$ 920.87, de acordo com AOAC (HORWITZ, 2000); o teor de lipídeos, pelo método n. ${ }^{\circ}$ 920.39, determinado por extração Soxhlet, de acordo com AOAC (CUNNIFF, 1995); o teor de cinza foi analisado pelo método n. ${ }^{\circ}$ 923.03, de acordo com AOAC (HORWITZ, 2000). A fibra alimentar total foi determinada pelo método n. ${ }^{\circ}$ 962.09, por digestão do material em solução de $\mathrm{H}_{2} \mathrm{SO}_{4}$ a $1,25 \%$ p/v por 30 minutos, seguida de $\mathrm{NaOH} 1,25 \% \mathrm{~m} / \mathrm{v}$ por mais 30 minutos, de acordo com AOAC (HORWITZ, 2000). Outros carboidratos foram obtidos por diferença.

Tabela 1. Formulações de pães de fôrma.

\begin{tabular}{|c|c|c|c|c|}
\hline \multirow[b]{2}{*}{ Ingredientes } & \multicolumn{4}{|c|}{ Formulações } \\
\hline & $\begin{array}{c}\text { Controle } \\
\text { (g) }\end{array}$ & $\begin{array}{c}10 \% \mathrm{FBV}^{*} \\
(\%)\end{array}$ & $\begin{array}{c}15 \% \text { FBV }^{*} \\
(\%)\end{array}$ & $\begin{array}{c}20 \% F^{*} V^{*} \\
(\%)\end{array}$ \\
\hline Farinha de Trigo Integral & 100 & 90 & 85 & 80 \\
\hline Farinha de Banana Verde & - & 10 & 15 & 20 \\
\hline Cloreto de Sódio & 2 & 2 & 2 & 2 \\
\hline Sacarose & 5 & 5 & 5 & 5 \\
\hline Fermento Biológico Fresco & 3 & 3 & 3 & 3 \\
\hline Ácido Ascórbico & 0,009 & 0,009 & 0,009 & 0,009 \\
\hline Água & $57-60$ & $57-60$ & $57-60$ & $57-60$ \\
\hline Gordura & 3 & 3 & 3 & 3 \\
\hline
\end{tabular}

*Em relação ao total de farinha de trigo integral. Fonte: El-Dash (1978), com modificações. 
É importante esclarecer que o método utilizado neste estudo para a determinação de fibras (método gravimétrico) determina fibra bruta; portanto, não se determina o conteúdo total de compostos que têm contribuição como fibra, sendo o resultado relacionado, quase que na totalidade, à fibra proveniente da farinha integral.

\subsubsection{Determinação do teor de AR das FBV e pães de fôrma integral}

O teor de AR foi determinado de acordo com o método da AOAC 996.11 (CUNNIFF, 1995), modificado por Walter et al. (2005), que recomenda a utilização de maior quantidade de amostra (300 mg em vez de $100 \mathrm{mg}$ ) e tampão fosfato pH 6,8, em vez de MOPS (ácido 4-morfolino propano sulfônico), $\mathrm{pH} 7,0$. As enzimas utilizadas para determinação de AR foram $\alpha$-amilase termoestável (Termamyl 2X), produzida pela Novozymes Latin American Limited (Araucária/Brasil), amiloglicosidase (Panzyn GA 400), produzida pela Prozyn (São Paulo/Brasil), e protease (Alcalase 2.4 L FG), produzida pela Novozymes Latin American Limited (Araucária/Brasil). A glicose liberada durante a hidrólise do amido foi determinada colorimetricamente $(\lambda=505 \mathrm{~nm})$, utilizando-se o kit glicose oxidase-peroxidase (Glicose PAP Liquiform, da Labtest). A quantidade de AR foi determinada de acordo com a Equação 3, descrita por Teixeira et al. (1998).

$A R=\frac{G \cdot 0,9 \cdot D \cdot 100}{P}$

em que: $A R=$ porcentagem de $A R$ em base seca; $G$ = concentração final de glicose $(\mathrm{mg} / \mathrm{mL})$; 0,9 = fator de conversão da glicose em amido; $D$ = fator de diluição da amostra; $\mathrm{P}=$ peso $(\mathrm{g})$ da amostra em base seca.

\subsubsection{Avaliação sensorial dos pães integrais adicionados de FBV}

A análise sensorial foi aprovada pelo Comitê de Ética da Universidade Federal do Rio Grande sob o número CAAE: 46815115.2.0000.5324 e foi realizada com 90 julgadores não treinados, escolhidos aleatoriamente entre alunos, professores e funcionários da instituição. As análises foram realizadas no Laboratório de Análise Sensorial da Universidade Federal do Rio Grande - Campus Carreiros. Os pães foram submetidos aos Testes Afetivos de Aceitação e Intenção de Compra (ABNT, 1993). Os testes foram aplicados no pão controle e nos pães adicionados de $15 \%$ de FBV, sendo esta formulação escolhida pelo maior teor de AR. Um Termo de Consentimento Livre e Esclarecido (TCLE) foi oferecido a cada avaliador, firmando o seu consentimento em participar da pesquisa.

O Índice de Aceitabilidade (IA) foi calculado conforme a Equação 4, descrita por Queiroz e Treptow (2006).
$\mathrm{IA} \%=\frac{\text { notamédia } \times 100}{9}$

\subsubsection{Análise estatística}

Para análise dos resultados das FBV, foi utilizada análise de variância one-way (ANOVA) com 95\% de significância $(\alpha), p \leq 0,05$; para os resultados da avaliação de volume específico, perda por cocção, dureza, parâmetros de cor da crosta, parâmetros de cor do miolo, composição proximal e AR dos pães de fôrma integral, foi também aplicada análise de variância one-way (ANOVA) com $95 \%$ de significância $(\alpha), p \leq 0,05$ e as médias obtidas foram comparadas pelo teste de Tukey utilizando o software Statistica 5.0 (StatSoft, Tulsa, OK, USA).

\section{Resultados e discussão}

\subsection{Composição proximal das farinhas}

O teor de umidade encontrado para as FBV das variedades Prata e Caturra foram, respectivamente, $6,3 \%$ e $6,6 \%$, valores que estão dentro dos padrões exigidos pela RDC 263/2005, que estabelece um teor máximo de umidade de 15\% para farinhas obtidas de frutos e sementes (BRASIL, 2005).

A caracterização das FBV das variedades Prata (FBV Prata) e Caturra (FBV Caturra), quanto à composição proximal, está apresentada na Tabela 2. Foi possível observar que a FBV Caturra apresenta maiores valores de proteínas e cinzas $(p>0,05)$, enquanto que, para lipídeos e fibras, não foi observada diferença significativa $(p>0,05)$ entre os valores. A FBV Prata se destacou no teor de carboidratos.

Quando comparamos os valores encontrados neste estudo com os encontrados na literatura, pode-se constatar que o teor de proteína encontrado para a FBV Prata está inferior ao encontrado por Borges et al. (2009), que obtiveram um valor de 4,7\% para a FBV Prata. O teor de proteína reportado por Fasolin et al. (2007) da FBV Caturra foi de 4,5\%, próximo ao encontrado neste estudo.

Quanto ao teor de lipídios, foi encontrado 0,3\% para banana Prata, valor este inferior ao encontrado por Santos et al. (2010), que relataram valor de 0,6\%. Em relação à FBV da

Tabela 2. Composição proximal das FBV em base seca.

\begin{tabular}{ccc|} 
Componentes & \multicolumn{2}{c|}{ Variedades } \\
\cline { 2 - 3 } & Prata (\%) & Caturra (\%) \\
\hline Proteína $^{1}$ & $3,0 \pm 0,99^{\mathrm{b}}$ & $5,2 \pm 0,03^{\mathrm{a}}$ \\
Lipídios $^{1}$ & $0,3 \pm 0,4^{\mathrm{a}}$ & $0,4 \pm 0,15^{\mathrm{a}}$ \\
Cinzas $^{1}$ & $2,2 \pm 0,02^{\mathrm{b}}$ & $3,0 \pm 0,14^{\mathrm{a}}$ \\
Fibras $^{1}$ & $0,6 \pm 0,18^{\mathrm{a}}$ & $0,6 \pm 0,06^{\mathrm{a}}$ \\
Carboidratos $^{2}$ & 94,5 & 91,4 \\
\hline
\end{tabular}

${ }^{1}$ Resultados expressos como média \pm desvio padrão de três determinações; ${ }^{2}$ Cálculo por diferença: 100 - (umidade + proteína + lipídios + cinzas). Em cada linha, médias seguidas de letras iguais não diferem significativamente entre si $(p>0,05)$. 
Produção de farinha de banana verde (Musa spp.) para aplicação em pão de trigo integral

Andrade, B. A. et al.

variedade Caturra, Torres et al. (2005) constataram um teor de $0,5 \%$.

Em relação às cinzas, o valor encontrado para a variedade Prata foi de $2,2 \%$, sendo este próximo ao encontrado por Borges et al. (2009). Para a variedade Caturra, Torres et al. (2005) obtiveram 2,0\% de cinzas em seu estudo com FBV, valor inferior ao encontrado neste trabalho.

Para as fibras, o valor encontrado foi 0,6\% para a FBV Prata, o qual é inferior ao verificado por Borges et al. (2009), que encontraram o dobro (1,2\%). Em relação à FBV Caturra, Medeiros et al. (2010) constataram um teor de fibras de $0,5 \%$, valor este semelhante ao verificado no presente estudo.

Portanto, podemos observar que, para a FBV Prata, os valores de lipídeos e fibras ficaram abaixo dos valores encontrados em literatura; já para a FBV Caturra, todos os valores ficaram superiores aos encontrados nesta consulta de dados. Existem fatores que influenciam nestes valores, como condições climáticas, qualidade de solo e disponibilidade de água; portanto, variações são aceitáveis.

\subsection{Rendimento das farinhas}

O rendimento da FBV para banana Prata foi de $26,4 \%$. Esse resultado foi próximo ao encontrado por Santos et al. (2010), que produziram FBV variedade Prata em secador de bandeja industrial a $40{ }^{\circ} \mathrm{C}$, por aproximadamente 24 horas, e encontraram 29,8\%. Para a FBV variedade Caturra, foi obtido um rendimento de 18,9\%, valor inferior ao encontrado por Fasolin et al. (2007), que verificaram um rendimento de $33,9 \%$.

\subsection{Teor de AR das farinhas}

O teor de AR nas farinhas das variedades Prata e Caturra foi de $24,1 \%$ e $13,7 \%$, respectivamente. De acordo com Juarez-Garcia et al. (2006), a FBV contém aproximadamente $24 \%$ de AR. Ramos et al. (2009) avaliaram farinhas de diferentes genótipos de banana verde, cujo procedimento para a obtenção das farinhas foi semelhante ao do presente trabalho; porém, a metodologia utilizada para a determinação do teor de AR foi a proposta por
Goñi et al. (1996), obtendo-se 20,4\% para a cultivar Prata Anã e 10,4\% para a cultivar Nanicão. As variações encontradas podem ser explicadas pelos diferentes métodos empregados em ambos os estudos.

\subsection{Avaliação da aplicação da FBV em pão de fôrma integral}

A Tabela 3 apresenta os resultados de volume específico, perda por cocção e dureza dos pães de fôrma integral controle e substituídos por FBV.

Foi possível observar que uma substituição de até $10 \%$ de farinha integral por FBV não alterou o volume específico do padrão; porém, foi observada uma mudança na textura, ficando esse pão mais duro. Tal condição significa que uma substituição de até $10 \%$ de farinha integral por FBV não interferiu no aprisionamento do gás dentro da massa e, portanto, no crescimento do pão.

A adição de FBV em 10\%, 15\% e 20\% aumentou a dureza, resultando em pães com massas não tão macias como a do pão controle. A adição de FBV influenciou mais a característica de textura do pão do que o volume específico, pois, na avaliação da dureza dos pães, todos os dados diferiram significativamente entre si $(p<0,05)$, com durezas maiores para os pães com maior concentração de FBV.

A diminuição do volume específico e o aumento da dureza estão associados à diluição que o glúten sofre, ou seja, o enfraquecimento da estrutura proteica (SEGUNDO et al., 2017; PAZ et al., 2015; FIGUEIRA et al., 2011).

Os valores de perda por cocção das amostras não diferiram significativamente $(p>0,05)$ entre estas. Ou seja, para a formulação de pão integral, não foi observada alteração da perda por cocção com a utilização da FBV na formulação. De acordo com Huerta (2015), as perdas durante a cocção devem-se à evaporação de líquidos, principalmente a água. Portanto, quanto menor a perda de cocção, maior o rendimento do pão.

Na Tabela 4, estão apresentados os parâmetros de cor do miolo e da crosta dos pães de fôrma integral controle e com 10, 15 e $20 \%$ de FBV.

Tabela 3. Volume específico, perda por cocção e dureza dos pães de fôrma integral controle e com FBV.

\begin{tabular}{cccc} 
Amostra & $\begin{array}{c}\text { Volume especifico } \\
(\mathbf{m L} / \mathbf{g})\end{array}$ & $\begin{array}{c}\text { Perda por cocção } \\
\text { Dureza }\end{array}$ & (N) \\
\hline Controle $^{1}$ & $2,6 \pm 0,16^{\mathrm{a}}$ & $8,2 \pm 0,62^{\mathrm{a}}$ & $8,4 \pm 0,21^{\mathrm{d}}$ \\
$10 \% \mathrm{FBV}^{1}$ & $2,5 \pm 0,13^{\mathrm{a}}$ & $8,6 \pm 0,44^{\mathrm{a}}$ & $12,2 \pm 0,21^{\mathrm{c}}$ \\
$15 \% \mathrm{FBV}^{1}$ & $2,0 \pm 0,07^{\mathrm{b}}$ & $8,9 \pm 0,84^{\mathrm{a}}$ & $15,1 \pm 0,34^{\mathrm{b}}$ \\
20\% FBV & $1,8 \pm 0,09^{\mathrm{b}}$ & $8,2 \pm 0,87^{\mathrm{a}}$ & $17,0 \pm 0,32^{\mathrm{a}}$ \\
\hline
\end{tabular}

${ }^{1}$ Resultados expressos como média \pm desvio padrão de três determinações. Em cada coluna, médias seguidas de letras iguais não diferem significativamente entre si $(p>0,05)$. 
Produção de farinha de banana verde (Musa spp.) para aplicação em pão de trigo integral

Andrade, B. A. et al.

Analisando-se a Tabela 4, verificou-se que os valores obtidos para a luminosidade $\left(L^{*}\right)$ para todos os pães apresentaram diferença significativa $(p<0,05)$ entre si. Foi observado que a substituição de FBV em 10\%, $15 \%$ e $20 \%$ contribuiu para o escurecimento do miolo e o clareamento da crosta do pão. Podemos observar ainda que o miolo mais escuro foi o dos pães com 10\% FBV e a crosta mais escura, a dos pães padrão, seguidos dos pães com 10\% e 20\% FBV.

Assim como a farinha integral, a FBV também contribui para a coloração do pão, pois, em relação à cromaticidade $a^{*}$, os pães com adição de FBV diferiram significativamente $(p<0,05)$ do pão controle, sendo o mesmo observado para a cromaticidade $b^{*}$. De forma geral, a adição de FBV reduziu os valores de $a^{*}$ e $b^{*}$, o que significa uma diminuição dos chromas vermelho e amarelo, respectivamente.

Um aspecto interessante foi investigado no estudo de Savlak et al. (2016), no qual eles observaram que a distribuição do tamanho das partículas afeta significativamente a cor das FBV, de forma que os valores de $L^{*}, a^{*}$ e b* decrescem com o aumento do tamanho da partícula $\left(r^{2}=0,94 ; r^{2}=0,72 ; r^{2}=0,73\right.$, respectivamente). Segundo Ahmed et al. (2015), o aumento dos valores de $L^{*}$ com a redução do tamanho das partículas é devido ao aumento da área superficial, levando a uma reflexão da luz. A utilização de partículas menores poderia trazer melhores resultados físicos e sensoriais do que os verificados no presente estudo.

\subsubsection{Composição proximal dos pães}

A caracterização dos pães de fôrma integrais controle e com 10, 15 e 20\% de FBV, quanto à composição proximal, está apresentada na Tabela 5.

Comparando-se o resultado de umidade do pão controle e dos pães com FBV, verificaram-se valores significativamente maiores para os pães com FBV. A maior absorção da FBV em relação à farinha integral pode estar associada ao maior teor de umidade dos pães com FBV.

O teor de proteína encontrado neste trabalho para o pão controle foi de $11,9 \%$, valor próximo ao encontrado por Fernandes (2006), de 12,9\%. Nota-se que as formulações com adição de FBV apresentaram menor teor de proteína em relação ao controle, o que se deve ao fato de a FBV apresentar um menor teor de proteínas do que a farinha de trigo; esse decréscimo de proteína também foi reportado por Ormenese (2010) e Silva et al. (2014).

Em relação ao teor de lipídios, os valores foram estatisticamente iguais.

Quanto às cinzas e fibras, não houve diferença significativa entre as amostras. O teor de carboidratos também não diferiu entre as amostras, o que pode ter sido devido à substituição de farinha integral por FBV em proporções pequenas, não sendo estas suficientes para o aumento e enriquecimento dos pães em cinzas, fibras e carboidratos.

Tabela 4. Parâmetros de cor do miolo e da crosta dos pães de fôrma integral controle e com de FBV.

\begin{tabular}{|c|c|c|c|c|}
\hline Parâmetro & Controle & $10 \%$ FBV & $15 \% \mathrm{FBV}$ & $20 \%$ FBV \\
\hline $\mathrm{L}^{*}(\text { miolo })^{1}$ & $59,94 \pm 0,53^{a}$ & $56,31 \pm 0,35^{c}$ & $57,47 \pm 0,38^{b}$ & $57,30 \pm 0,35^{b}$ \\
\hline$a^{*}(\text { miolo })^{1}$ & $+6,32 \pm 0,29 a$ & $+5,28 \pm 0,16^{b}$ & $+5,48 \pm 0,25^{b}$ & $+5,35 \pm 0,17^{b}$ \\
\hline$b^{\star}(\text { miolo })^{1}$ & $+18,82 \pm 0,13^{a}$ & $+17,47 \pm 0,28^{b}$ & $+17,47 \pm 0,22^{b}$ & $+17,23 \pm 0,28^{b}$ \\
\hline$L^{*}(\text { crosta })^{1}$ & $59,49 \pm 1,35^{c}$ & $61,82 \pm 0,44^{b}$ & $64,56 \pm 0,25^{a}$ & $62,25 \pm 0,69^{b}$ \\
\hline$a^{\star}(\text { crosta })^{1}$ & $12,82 \pm 0,58^{a}$ & $7,88 \pm 0,36^{b}$ & $5,51 \pm 0,45^{c}$ & $8,29 \pm 1,02^{b}$ \\
\hline$b^{*}(\text { crosta })^{1}$ & $30,77 \pm 0,64^{a}$ & $26,96 \pm 0,65^{b}$ & $21,05 \pm 0,96^{c}$ & $29,47 \pm 0,89^{a}$ \\
\hline
\end{tabular}

${ }^{1}$ Resultados expressos como média \pm desvio padrão de cinco determinações. Em cada linha, médias seguidas de letras iguais não diferem significativamente entre si $(p>0,05)$.

Tabela 5. Composição proximal do pão integral controle e dos pães com de FBV.

\begin{tabular}{|c|c|c|c|c|c|c|c|c|}
\hline \multirow{3}{*}{ Componentes } & \multicolumn{8}{|c|}{ Pães } \\
\hline & \multicolumn{2}{|c|}{ Controle } & \multicolumn{2}{|c|}{ FBV $10 \%$} & \multicolumn{2}{|c|}{ FBV $15 \%$} & \multicolumn{2}{|c|}{ FBV $20 \%$} \\
\hline & b.s. & b.u. & b.s. & b.u. & b.s. & b.u. & b.s. & b.u. \\
\hline Umidade $^{1}$ & - & $38,8 \pm 0,08^{c}$ & - & $40,5 \pm 0,22^{b}$ & - & $41,5 \pm 0,44^{a}$ & - & $42,1 \pm 0,24^{a}$ \\
\hline Proteína ${ }^{1}$ & $11,9 \pm 1,50^{a}$ & $7,3 \pm 0,92^{a}$ & $11,3 \pm 0,27^{a}$ & $6,7 \pm 0,19 a b$ & $10,9 \pm 0,27^{a}$ & $6,4 \pm 0,16^{a b}$ & $10,1 \pm 0,30^{a}$ & $5,9 \pm 0,18^{b}$ \\
\hline Lipídios ${ }^{1}$ & $2,1 \pm 0,04^{a}$ & $1,3 \pm 0,03^{a}$ & $2,1 \pm 0,08^{a}$ & $1,3 \pm 0,04^{a}$ & $2,2 \pm 0,07^{a}$ & $1,3 \pm 0,04^{a}$ & $2,2 \pm 0,04^{a}$ & $1,3 \pm 0,02^{a}$ \\
\hline Cinzas $^{1}$ & $3,5 \pm 0,03^{c}$ & $2,1 \pm 0,02^{b}$ & $3,6 \pm 0,03^{b}$ & $2,2 \pm 0,01^{a b}$ & $3,6 \pm 0,02^{b}$ & $2,1 \pm 0,01^{a b}$ & $3,8 \pm 0,04^{a}$ & $2,22 \pm 0,01^{a}$ \\
\hline Fibras $^{1}$ & $2,1 \pm 0,09^{a}$ & $1,3 \pm 0,05^{a}$ & $2,0 \pm 0,2^{a}$ & $1,2 \pm 0,12^{a}$ & $1,9 \pm 0,21^{a}$ & $1,1 \pm 0,13^{a}$ & $1,9 \pm 0,05^{a}$ & $1,1 \pm 0,04^{a}$ \\
\hline Carboidratos $^{2}$ & 82,5 & 50,5 & 83,0 & 49,3 & 83,3 & 48,7 & 83,9 & 48,5 \\
\hline
\end{tabular}

${ }^{1}$ Resultados expressos como média \pm desvio padrão de três determinações; Cálculo por diferença: 100 - (umidade + proteína + lipídios + cinzas). Em cada linha, médias seguidas de letras iguais não diferem significativamente entre si $(p>0,05)$. 
Produção de farinha de banana verde (Musa spp.) para aplicação em pão de trigo integral

Andrade, B. A. et al.

\subsubsection{Teor de AR dos pães integrais}

O teor de AR dos pães controle e com substituição de FBV encontra-se na Tabela 6.

Os pães de 15 e $20 \%$ de FBV apresentaram teor de AR significativamente $(p<0,05)$ superior ao das demais formulações. Ormenese (2010) encontrou diferença significativa entre os pães de 10 e $20 \%$ de substituição de FBV.

\subsection{Avaliação sensorial dos pães de fôrma integral controle e com $15 \%$ de FBV}

Foi possível observar através dos resultados que os pães com $15 \%$ e $20 \%$ de FBV apresentaram teores de AR significativamente $(p<0,05)$ superiores aos das demais formulações, e que esses resultados não diferiram entre si; note-se que a dureza do pão com $20 \%$ foi significativamente maior. Considerando-se o volume específico e a dureza, que são parâmetros muito importantes para a aceitação sensorial, foi escolhida a formulação com $15 \%$ para a avaliação sensorial.

Tabela 6. Teor de amido resistente (AR) do pão integral controle e dos pães com FBV.

\begin{tabular}{ccc} 
Formulações & AR (\%) & Aumento de AR (\%) \\
Controle $^{1}$ & $10,27 \pm 1,10^{\mathrm{b}}$ & - \\
$10 \% \mathrm{FBV}^{1}$ & $10,88 \pm 0,95^{\mathrm{b}}$ & 5,94 \\
$15 \% \mathrm{FBV}^{1}$ & $16,26 \pm 1,28^{\mathrm{a}}$ & 58,33 \\
$20 \% \mathrm{FBV}^{1}$ & $16,91 \pm 0,84^{\mathrm{a}}$ & 64,65 \\
\hline
\end{tabular}

${ }^{1}$ Resultados expressos como média \pm desvio padrão de três determinações. Na coluna, médias seguidas de letras iguais não diferem significativamente entre si $(p>0,05)$.
A classificação dos escores hedônicos dos pães de fôrma integral controle e 15\% FBV, para os atributos cor, aroma, sabor e textura, está apresentada na Figura 1.

Observando-se o atributo cor, é notável que o pão controle obteve um percentual expressivo de avaliadores, que atribuíram a nota 9, nota máxima da escala hedônica, definida por "Gostei muitíssimo"; somando-se ao percentual de avaliadores que atribuíram notas 7 e 8 , totalizaram-se $90 \%$ dos avaliadores, sendo que apenas $10 \%$ atribuíram notas 5 ou 6 . Não foi observada nenhuma nota menor ou igual a 4 . Os pães com $15 \%$ de FBV obtiveram um percentual menor de notas maiores ou iguais a $7(73,3 \%)$, o dobro de notas 5 e $6(20 \%)$ e $6,7 \%$ dos avaliadores atribuíram notas menores ou iguais a 4 , notas com conceito definidos dentro de "Desgostei". Ormenese (2010), em seu estudo, também observou que, para o requisito aparência, quanto maior o percentual de FBV, menores foram as médias obtidas dos avaliadores.

Os resultados para o atributo sabor também apresentaram uma distribuição de notas muito semelhante à cor, inclusive com percentuais bem próximos. O sabor é um requisito importante para o consumidor, sendo observada, neste estudo, influência da substituição de farinha de banana verde sobre esse atributo.

$\mathrm{O}$ atributo menos influenciado pela adição de FBV foi o aroma, com uma classificação de escores muito próximos. O mesmo foi observado por Carvalho et al. (2012), que desenvolveram cupcake adicionado de farinha da casca de banana.

Para o atributo textura, as notas ficaram distribuídas apenas com percentuais iguais para as notas menores ou iguais a 4. Este atributo pode ter influenciado a aceitação
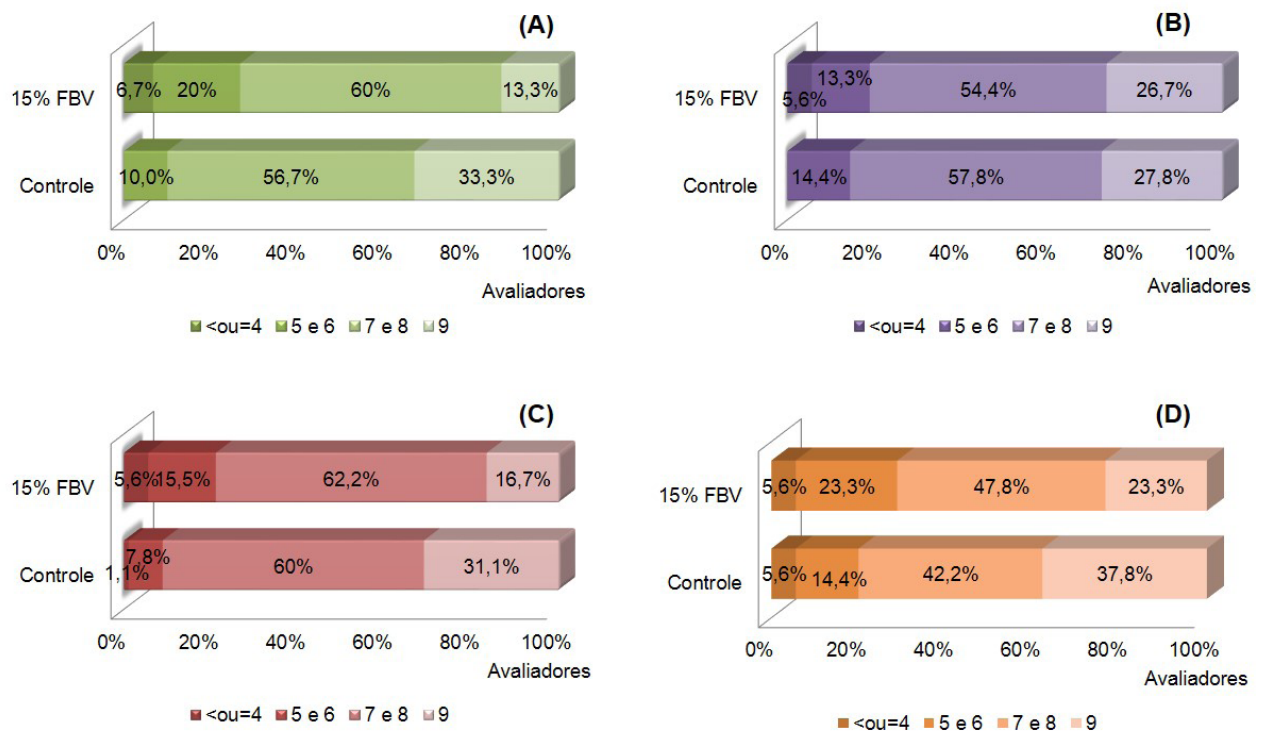

Figura 1. Classificação dos escores hedônicos para os atributos cor (A), aroma (B), sabor (C) e textura (D) dos pães de fôrma integrais controle e $15 \%$ de FBV. 
Produção de farinha de banana verde (Musa spp.) para aplicação em pão de trigo integral

Andrade, B. A. et al.

dos consumidores, pois a média foi menor tanto para o pão controle como para o pão de 15\% de FBV.

O estudo realizado por Segundo et al. (2017) mostrou que FBV pode ser usada para melhorar as propriedades nutricionais de bolos em camadas sem afetar negativamente suas propriedades físicas e resultando em um pequeno declínio na percepção sensorial. Por outro lado, os atributos físicos e sensoriais de pão de ló foram notoriamente piorados com o uso da FBV.

Os pães controle e adicionados de $15 \%$ de FBV atingiram Índices de Aceitação (IA) de 88,7\% e 82,1\%; segundo Queiroz e Treptow (2006), para ser considerado aceito pelos consumidores, um produto salgado deve ter um mínimo de aceitação de $70 \%$. A diferença entre os valores de IA encontrados está associada principalmente aos atributos cor e sabor do pão.

Em relação aos resultados da intenção de compra, observou-se uma atitude positiva na compra dos pães controle e com FBV. Para o pão controle, $70 \%$ dos avaliadores "Comprariam frequentemente" $(46,7 \%)$ ou "Comprariam sempre" (23,3\%); para o pão com 15\% de FBV, aproximadamente $56 \%$ dos avaliadores "Comprariam frequentemente" ( $41,1 \%)$ ou "Comprariam sempre" (14,4\%).

\section{Conclusão}

A farinha de banana verde (FBV) que apresentou o maior teor de amido resistente (AR) foi a da variedade Prata. Comparando-se os pães, observou-se que a substituição de farinha de trigo integral por FBV afetou o volume específico e a dureza dos pães. Uma substituição de $10 \%$ de farinha integral por FBV não alterou o volume específico, porém foi observada uma mudança significativa na dureza do pão. A substituição de FBV em 10\%, 15\% e 20\% aumentou significativamente a dureza dos pães, resultando em pães com massas não tão macias como a do pão controle. A adição de FBV influenciou mais a característica de textura do pão do que do volume específico. Com relação à cor, a substituição de FBV em $10 \%, 15 \%$ e $20 \%$ contribuiu para o escurecimento do miolo e o clareamento da crosta do pão. Os pães controle e adicionados de 15\% FBV atingiram Índices de Aceitação Sensorial de 88,7\% e 82,1\%, respectivamente. Apesar de observarmos um aumento na dureza dos pães adicionados de FBV, a diferença dos valores de índice de aceitação está associada principalmente aos atributos cor e sabor do pão. A atitude de compra dos consumidores foi positiva e não foi observada rejeição para nenhuma das formulações de pão testadas.

\section{Referências}

AHMED, J.; AL-JASSAR, S.; THOMAS, L. A comparison in rheological, thermal, and structural properties between Indian Basmati and Egyptian Giza rice flour dispersions as influenced by particle size. Food Hydrocolloids, v. 48, p. 72-83, 2015. http://dx.doi.org/10.1016/j.foodhyd.2015.02.012.

AMERICAN ASSOCIATION OF CEREAL CHEMIST - AACC. Approved methods of the AACC. 10th ed. St. Paul: AACC, 2000. ASSOCIAÇÃO BRASILEIRA DE NORMAS TÉCNICAS - ABNT. NBR 1994: análise sensorial dos alimentos e bebidas. Rio de Janeiro, 1993. p. 2.

BORGES, A. M.; PEREIRA, J.; LUCENA, E. M. P. Caracterização da farinha de banana verde. Ciência e Tecnologia de Alimentos, v. 29, n. 2, p. 333-339, 2009. http://dx.doi.org/10.1590/S010120612009000200015.

BRASIL. Ministério da Saúde. Agência Nacional de Vigilância Sanitária - ANVISA. Resolução $n^{\circ} 263$, de 22 de setembro de 2005. Dispõe sobre o regulamento técnico para produtos de cereais, amidos, farinhas e farelos. Diário Oficial [da] República Federativa do Brasil, Brasília, DF, 22 set. 2005. Seção 1.

CARVALHO, K. H.; BOZATSKI, L. C.; SCORSIN, M.; NOVELLO, D.; PEREZ, E.; DALLA SANTA, H. S.; SCORSIN, G.; BATISTA, M. $G$. Desenvolvimento de cupcake adicionado de farinha da casca de banana: características sensoriais e químicas. Alimentos e Nutrição, v. 23, n. 3, p. 475-481, 2012.

CHAMP, M.; FAISANT, N. Resistant starch: analytical and physiological aspects. Boletim da SBCTA, v. 30, n. 1, p. 37-43, 1996.

CHITARRA, M. I. F. E.; CHITARRA, A. B. Pós-colheita de frutas e hortaliças: fisiologia e manuseio. Lavras: Editora UFLA, 2005. $785 \mathrm{p}$.

COELHO JÚNIOR, L. M. C. Concentração regional do valor de produção da banana do Paraná, Brasil (1995 a 2010). Ciência Rural, v. 43, n. 12, p. 2304-2310, 2013. http://dx.doi.org/10.1590/ S0103-84782013005000137.

CUNNIFF, P. (Ed.) Official methods of analysis of the Association of Official Analytical Chemists. 16th ed. Washington: AOAC, 1995.

EL-DASH, A. A. Standardized mixing and fermentation procedure for experimental baking test. Cereal Chemistry, v. 55, n. 4, p. 436-446, 1978.

FASOLIN, L. H.; ALMEIDA, G. C.; CASTANHO, P. S.; NETTOOLIVEIRA, E. R. Biscoitos produzidos com farinha de banana: avaliações química, física e sensorial. Ciência e Tecnologia de Alimentos, v. 27, n. 3, p. 524-529, 2007. http://dx.doi.org/10.1590/ S0101-20612007000300016.

FERNANDES, A. F. Utilização da farinha de casca de batata inglesa (Solanum tuberosum L.) na elaboração de pão integral. 2006. 127 f. Dissertação (Mestrado)-Programa de Pós-graduação em Ciências dos Alimentos, Universidade Federal de Lavras, Lavras, 2006.

FIGUEIRA, F. S.; CRIZEL, T. M.; SILVA, C. R.; SALAS-MELLADO, M. M. Pão sem glúten enriquecido com a microalga Spirulina 
Produção de farinha de banana verde (Musa spp.) para aplicação em pão de trigo integral Andrade, B. A. et al.

platensis. Brazilian Journal of Food Technology, v. 14, n. 4, p. 308-316, 2011. http://dx.doi.org/10.4260/BJFT2011140400037.

GOÑI, I.; GARCÍA-DIZ, L.; MANÃS, E.; SAURA-CALIXTO, F. Analysis of resistant starch: a method for foods and food products. Food Chemistry, v. 56, n. 4, p. 445-449, 1996. http://dx.doi. org/10.1016/0308-8146(95)00222-7.

HORWITZ, W. (Ed.). Official methods of analysis of the Association of Official Analytical Chemists. 17th ed. Washington: AOAC, 2000

HUERTA, K. M. Utilização de farinha de chia (Salvia hispânica L.) na elaboração de pão sem glúten sem adição de goma e gordura. 2015. 117 f. Dissertação (Mestrado)-Programa de Pós-graduação em Ciências e Tecnologia de Alimentos, Universidade Federal de Santa Maria, Santa Maria, 2015.

INSTITUTO BRASILEIRO DE GEOGRAFIA E ESTATÍSTICA - IBGE. Pesquisa mensal de previsão e acompanhamento das safras agrícolas no ano civil. Levantamento Sistemático da Produção Agrícola, v. 29, n. 2, p. 1-81, 2016.

JUAREZ-GARCIA, E.; AGAMA-ACEVEDO, E.; SAYAGO-AYERDI, S. G.; RODRIGUEZ-AMBRIZ, S. L.; BELLO-PEREZ, L. A. Composition, digestibility and application in breadmaking of banana flour. Plant Foods for Human Nutrition (Dordrecht, Netherlands), v. 61, n. 3, p. 131-137, 2006. PMid:17048100. http://dx.doi.org/10.1007/ s11130-006-0020-x.

LANGKILDE, A. M.; CHAMP, M.; ANDERSSON, H. Effects of highresistant-starch banana flour (RS2) on in vitro fermentation and the small-bowel excretion of energy, nutrients, and sterols: an ileostomy study. The American Journal of Clinical Nutrition, v. 75 , n. 1, p. 104-111, 2002. PMid:11756067.

MEDEIROS, M. J.; OLIVEIRA, P. A. A. C.; SOUZA, J. M. L.; SILVA, R. F.; SOUZA, M. L. Composição química de misturas de farinhas de banana verde com castanha-do-brasil. Revista do Instituto Adolfo Lutz, v. 69, n. 3, p. 396-402, 2010.

MEDINA, V. M.; PEREIRA, M. E. C. Pós-colheita. In: BORGES, A. L.; SOUZA, L. S. O cultivo da bananeira. Cruz das Almas: EMBRAPA, 2004. cap. 12. 219 p.

MINOLTA CORPORATION. Precise color communication: color control from feeling to instrumentation. Ramsey: Minolta Corporation Instrument Systems Division, 1994. p. 49.

ORMENESE, R. C. S. C. Obtenção de farinha de banana verde por diferentes processos de secagem e aplicação em produtos alimentícios. 2010. 182 f. Tese (Doutorado em Tecnologia de Alimentos)-Faculdade de Engenharia de Alimentos, Universidade Estadual de Campinas, Campinas, 2010.

PAZ, M. F.; MARQUES, R. V.; SCHUMANN, C.; CORRÊA, L. B.; CORREAA, E. K. Características tecnológicas de pães elaborados com farelo de arroz desengordurado. Brazilian Journal of Food Technology, v. 18, n. 2, p. 128-136, 2015. http://dx.doi. org/10.1590/1981-6723.6014.
PEREIRA, K. D. Amido resistente, a última geração no controle de energia e digestão saudável. Ciências e Tecnologia de Alimentos, v. 27, p. 88-92, 2007. http://dx.doi.org/10.1590/ S0101-20612007000500016.

PIZZINATTO, A.; MAGNO, C. P. R. S.; CAMPAGNOLLLI, D. M. F.; VITTI, P.; LEITÃO, R. F. F. Avaliação tecnológica de produtos derivados de farinha de trigo (pão, macarrão, biscoito). 3 . ed. Campinas: ITAL, 1993. p. 54

PRILL, M. A. S.; NEVES, L. C.; CHAGAS, E. A.; TOSIN, J. M.; SILVA, S. S. Atmosfera modificada e controle de etileno para bananas 'Prata-Anã' cultivadas na Amazônia Setentrional Brasileira. Revista Brasileira de Fruticultura, v. 34, n. 4, p. 1030-1042, 2012. http://dx.doi.org/10.1590/S0100-29452012000400009.

QUEIROZ, M. I.; TREPTOW, R. O. Análise sensorial para avaliação da qualidade dos alimentos. Rio Grande: Ed. FURG, 2006. 268 p.

RAMOS, D. P.; LEONEL, M.; LEONEL, S. Amido resistente em farinhas de banana verde. Alimentos e Nutrição, v. 20, n. 3, p. 479-483, 2009

SANTOS, J. C.; SILVA, G. F.; SANTOS, J. A. B.; OLIVEIRA JÚNIOR, A. M. Processamento e avaliação da estabilidade da farinha de banana verde. Exacta, v. 8, n. 2, p. 219-224, 2010.

SARAWONG, C.; SCHOENLECHNER, R.; SEKIGUCHI, K.; BERGHOFER, E.; NG, P. K. Effect of extrusion cooking on the physicochemical properties, resistant starch, phenolic content and antioxidant capacities of green banana flour. Food Chemistry, v. 143, p. 33-39, 2014. PMid:24054209. http://dx.doi.org/10.1016/j. foodchem.2013.07.081.

SAVLAK, N.; TÜRKER, B.; YESILKANAT, N. Effects of particle size distribution on some physical, chemical and functional properties of unripe banana flour. Food Chemistry, v. 213, p. 180-186, 2016. PMid:27451170. http://dx.doi.org/10.1016/j. foodchem.2016.06.064.

SEGUNDO, C.; ROMÁN, L.; GÓMEZ, M.; MARTINEZ, M. M. Mechanically fractionated flour isolated from green bananas (M. cavendishii var. nanica) as a tool to increase the dietary fiber and phytochemical bioactivity of layer and sponge cakes. Food Chemistry, v. 219, p. 240-248, 2017. PMid:27765223. http:// dx.doi.org/10.1016/j.foodchem.2016.09.143.

SILVA, A. A.; BARBOSA JUNIOR, J. L.; BARBOSA, M. I. M. J. Farinha de banana verde como ingrediente funcional em produtos alimentícios. Ciência Rural, v. 45, n. 12, p. 2252-2258, 2015. http://dx.doi.org/10.1590/0103-8478cr20140332.

SILVA, J. P.; NETTO-OLIVEIRA, E. R.; PEREIRA, S. C. M.; MONTEIRO, A. R. G. Avaliação físico-química e sensorial de pães produzidos com substituição parcial de farinha de trigo por farinha de banana verde. Revista Brasileira de Pesquisa em Alimentos, v. 5, n. 3, p. 1-7, 2014.

SOUZA, M. E.; LEONEL, S.; FRAGOSO, A. M. Crescimento e produção de genótipos de bananeiras em clima subtropical. 
Produção de farinha de banana verde (Musa spp.) para aplicação em pão de trigo integral

Andrade, B. A. et al.

Ciência Rural, v. 41, n. 4, p. 581-591, 2011. http://dx.doi. org/10.1590/S0103-84782011005000032.

TEIXEIRA, M. A. V.; CIACCO, C. F.; TAVARES, D. Q.; BONEZZI, A. N. Ocorrência e caracterização do amido resistente em amidos de milho e de banana. Ciência e Tecnologia de Alimentos, v. 18, n. 2, p. 246, 1998. http://dx.doi.org/10.1590/S010120611998000200019.

TORRES, L. L. G.; EL-DASH, A. A.; CARVALHO, C. W. P.; ASCHERI, J. L. R.; GERMANI, R.; MIGUEZ, M. Efeito da umidade e da temperatura no processamento de farinha de banana verde (Musa acuminata, grupo AAA) por extrusão termoplástica. Boletim do Centro de Pesquisa de Processamento de Alimentos, v. 23, n. 2, p. 273-290, 2005. http://dx.doi.org/10.5380/cep.v23i2.4488. WALTER, M.; SILVA, L. P.; PERDOMO, D. M. X. Amido disponível e resistente em alimentos: adaptação do método da AOAC 996.11. Alimentos e Nutrição, v. 16, n. 1, p. 39-43, 2005.

YUE, P.; WARING, S. Resistant starch in food applications. Cereal Foods World, v. 43, n. 9, p. 690-695, 1998. 